Voix et Images

\title{
Comment découper le corps certain et s'en sortir avec passion. Entretien avec Roger Des Roches
}

\section{André Gervais}

Volume 13, numéro 2 (38), hiver 1988

Le propre du corps Roger Des Roches

URI : https://id.erudit.org/iderudit/200704ar

DOI : https://doi.org/10.7202/200704ar

Aller au sommaire du numéro

Éditeur(s)

Université du Québec à Montréal

ISSN

0318-9201 (imprimé)

1705-933X (numérique)

Découvrir la revue

Citer ce document

Gervais, A. (1988). Comment découper le corps certain et s'en sortir avec passion. Entretien avec Roger Des Roches. Voix et Images, 13(2), 217-244.

https://doi.org/10.7202/200704ar d'utilisation que vous pouvez consulter en ligne.

https://apropos.erudit.org/fr/usagers/politique-dutilisation/ 


\section{Comment découper le corps certain et s'en sortir avec passion'1 Entretien avec Roger Des Roches}

\section{par André Gervais, Université du Québec à Rimouski}

V. \& I. - Identifions d'abord les débuts, la «préhistoire»: comment cela commence-t-il à s'écrire, et ce, avant même le premier livre?

R. DES ROCHES - ...Comme si c'était normal... Je ne sais pas... Comme si ça allait de soi, sans poser de questions (déjà j'évitais - je cultivais ce réflexe qui m'évitait de me poser de trop grandes questions sur l'origine de mes gestes)... Comme si c'était parfaitement normal, naturel - non pas spontané, mais «naturel». Une vague fonction biologique. Le sport pour les autres, l'écriture pour moi: penché sur une feuille, avec des livres modèles, avec le désir d'écrire des livres avant même de savoir ce qu'était vraiment un livre - et un modèle...

Mes premiers textes - les premiers textes que je sens motivés par une volonté d'écrire relativement identifiable — datent de 1963, c'est-à-dire vers l'âge de 13 ans. J'étudie alors (et je n'étudie pas vraiment) au collège classique de Longueuil, en Syntaxe.

Voici donc les premiers textes qui ne traînent pas l'étiquette et le ton et la tristesse de la rédaction scolaire: ces «textes de la tâche», ces textes dirigés par la pauvre imagination des autres, ces textes que j'avais d'ailleurs, jusqu'à ce jour, laissés aux bons soins de ma mère (ma mère, ghost-writer sans le savoir)...

Mes premières productions «littéraires» venaient d'une espèce de désir... de désir criant - qui ne souffrait aucun retard —, impatient, de... faire naître quelque chose. Quoi au juste, je ne sais pas. Je ne sais toujours pas. Ce désir-là, je le ressens encore. Il fait toujours aussi mal quand ce sont les autres qui écrivent les livres que je devrais écrire... Il fallait que quelque chose naisse vite, très vite, fini, naisse bien, naisse beau, que quelque chose éclate de tous ces gestes bizarres exécutés en silence, sans trop savoir comment et pourquoi.

J'ai commencé par de très courts textes. Le souffle très court. Des textes fortement influencés par mes lectures distraites et imparfaites de Verlaine, de Rimbaud (de ce Rimbaud dont je n'aurai jamais apprécié que l'indécence et auquel, malgré tout ce que Charron a vainement tenté de m'y faire voir, je n'ai jamais accordé qu'une attention agacée — son talent ne m'a jamais paru évident, pas même aujourd'hui!).

1 L'entretien a eu lieu à Montréal, le 10 juillet 1987. Il a été relu et récrit par Roger Des Roches en novembre 1987. 
Ne sachant que dire, j'écrivais des textes... d'atmosphère. Des textes de sentiments - sans trop savoir ce qu'étaient les sentiments, bien sûr - où il fallait les placer - ce qu'il fallait leur faire subir pour qu'ils deviennent intéressants à raconter. Des textes «descriptifs», à peu près aussi rigoureux dans leur structure et dans leur logique que ces petits rêves idiots qu'on peut faire, en série, la nuit, lorsque même l'inconscient n'a plus grand chose d'important à dire...

Je lisais Verlaine, Rimbaud, Baudelaire... Asimov, Bradbury et Henri Vernes...

V. \& I. - Henri, pas Jules. Henri Vernes, l'auteur des «Bob Morane»...

R. DES ROCHES - Exact...

V. \& I. - Ça ne fait pas très sérieux dans le parcours d'un écrivain...

R. DES ROCHES - Un parcours d'écrivain n'est jamais quelque chose de particulièrement sérieux. Ou d'honnête. Il existe toujours plus de zones grises - les lectures idiotes, les naïvetés, de grands élans et d'égoïsmes - que de moments éclatants, que de merveilleuses intuitions.

Bob Morane, les livres de Maurice Limat et de F. Richard-Bessière dans la collection «Anticipation» de Fleuve Noir... La fascination que pouvaient exercer sur moi des titres comme les Chasseurs de dinosaures, Un parfum d'Ylang-Ylang, la Terre n'est pas ronde, Plus égale moins, Pas de Gonia pour les Gharkandes...

J'ai parfois l'impression d'avoir beaucoup plus appris à écrire en lisant Henri Vernes qu'en lisant Rimbaud. Non!... Laissez-moi corriger ce qui précède: je sais que j'ai plus appris à écrire en lisant Vernes que Rimbaud. Chez Henri Vernes, je découvrais le plaisir. Le plaisir à l'état pur. Le plaisir sans explications à donner. Sans excuses. Le plaisir de lire d'abord. Puis je découvrais - j'assimilais, sans me l'expliquer — que le plaisir de lire ressemblait sûrement, d'une certaine manière, au plaisir d'écrire, et que ce dernier ne pouvait se dissocier de la rigueur, de la couleur, de l'imagination.

Je commençais aussi, cette année-là, à lire en langue originale des livres de science-fiction américaine et anglaise, à suivre l'évolution de la science-fiction et du fantastique au cinéma et à la télévision. Je me découvrais ainsi ma première réelle passion de lecture. Sans vouloir faire un résumé trop hâtif de ma vie d'écrivain, je pourrais dire que j'ai eu trois grandes passions. Dans l'ordre: la science-fiction, la poésie... disons «expérimentale» (surtout surréaliste) et, aujourd'hui, l'horreur. Tout le reste, la théorie, le politique, n'auront été qu'une espèce de maquillage contextuel...

Mais remontons plus loin en arrière. Donc avant ces premiers «textes» de1962-1963. Je ne vois qu'une énorme difficulté à écrire: Un mur. Un autre monde. Comme si le geste même... Je me battais autant avec la forme des lettres qu'avec le contenu des mots. Comme si le geste même d'écrire était quelque chose d'incroyablement étranger... J'écrivais mal, mal dans tous les sens... Je ne voyais dans l'écriture qu'un devoir de plus (et moi, le devoir, ça me tue!), une obligation à comprendre et à me servir d'un mécanisme inutile... Je 
n'avais pas encore fait l'équation entre le livre écrit et le geste de l'écrire... Il me semble, à y songer aujourd'hui, que les livres que je lisais alors - et dans lesquels je me perdais avec plaisir — n'avaient tout simplement pas d'auteurs, ou que leurs auteurs ne l'étaient que par hasard, passivement, des prête-noms, que leurs livres, tout compte fait, étaient le fruit d'une espèce de génération spontanée. Des ready-made pour adolescents. Ce n'est donc qu'en faisant cette relation du plus bas niveau entre la lecture et l'écriture, puis en me découvrant un plaisir pervers à écrire, que j'ai pu faire les pas nécessaires pour devenir écrivain.

\section{V. \& I. - L'écriture, à cette époque, n'était pas votre seule préoccupation...}

R. DES ROCHES - Le dessin, la peinture. Des gestes plus clairs, plus évidents, plus... romantiques. Dès l'âge de 6 ou 7 ans, je me disais: «Un jour, je serai peintre, un jour, je serai un artiste.» Mais j'ai perdu l'intérêt. Maintenant, je ne sors plus mes pinceaux qu'une ou deux fois par année, for old times sake... Parfois je réalise deux ou trois illustrations, habituellement pour un ou une collègue en particulier, pour illustrer un recueil, par exemple.

V. \& I. - Vous concevez toujours les maquettes de couvertures de vos propres livres.

R. DES ROCHES - Toutes, en effet, sauf pour ce qui est de mes deux premiers ouvrages aux Éditions du Jour: Corps accessoires (1970) et l'Enfance d'yeux (1972).

V. \& I. - Et vous avez conçu des couvertures pour d'autres auteurs...

R. DES ROCHES - Oui, pour François Charron, Lucien Francœur, Claude Robitaille, Yolande Villemaire, Normand de Bellefeuille. J'ai préparé les maquettes de base de certains périodiques culturels: les Herbes rouges, Stratégie et Spirale. Mais là aussi j'ai perdu l'intérêt. D'ailleurs, dans les arts, je ne comprends plus vraiment ce qui s'y passe. Trop de temps, trop de styles ont passé. Mais j'ai perdu l'intérêt... Je sais ce que j'aime, mais je n'ai aucune idée si ça a aucune valeur. Jusqu'à tout récemment, j'ai produit de manière très irrégulière des encres servant à «illustrer» certains livres d'André Roy, de Lucie Ménard, de Normand de Bellefeuille. Là aussi, plus tellement d'intérêt. Plus d'intérêt, donc plus de recherche; plus de recherche, donc plus d'influences; plus d'influences, donc plus de talent...

Mais pendant que je perdais le goût des arts, je découvrais les livres qui devaient m'influencer, cette fois-ci en poésie. En 1965, je découvrais en même temps et de façon un peu anarchique les Essais rouges de Claude Péloquin, qui m'amena à Dada (les manifestes surtout), Je de Denis Vanier qui m'amena au Surréalisme, à l'Homme approximatif de Tristan Tzara, puis Prévert, puis Breton (sur le tard). Ceci venait s'ajouter à ce que j'avais glané à propos de la peinture cubiste, surréaliste, abstraite. Vers 1966-1967, lorsque je ne nourrissais pas quelque prétention à devenir un célèbre écrivain de science-fiction, j'écrivais de petits textes dadaïstes en vers, des espèces de petits manifestes dadaïstes en 
prose qui ne voulaient strictement rien dire. C'était d'ailleurs ma fine analyse du manifeste dadaïste: manifester quelque chose, peu importe, dans l'absolu, hors du sens - la preuve que j'ai toujours eu de grandes difficultés à faire sens d'un texte qui quittait le domaine familier de la fiction.

Mais malgré ces lectures, entre 1965 et 1968, je ne suis vraiment pas au courant de ce qui se fait ici, en général, en littérature. Je ne connais que Péloquin, Vanier et Piazza. Ces années sont beaucoup plus, pour moi, des années d'exploration, où je deviens une espèce de touche-à-tout naîf aux ambitions mal définies, multiples, et singulièrement contradictoires. Ma démarche devient un meltingpot de toutes sortes de littératures (peut-être déjà les premiers livres de Sade où je ne retiens sûrement pas le discours philosophique!). J'écris de tout. J'ai le goût de tout. Voici qui résume assez bien ma démarche initiale, mon apprentissage autodidacte du geste d'écrire. Je ne comprends pas nécessairement, mais j'ai le goût.

V. \& I. - Mais comment fait-on, à l'Externat classique de Longueuil (qui deviendra le cégep Edouard-Montpetit), puis au Secondaire, entre 12 et 16 ans, pour se brancher sur cette littérature qui n'est pas du tout la littérature enseignée?

R. DES ROCHES - Il faut se rappeler qu'à cette époque, il n'y avait pas de «littérature enseignée». Sûrement pas au Secondaire. On n'enseignait pas la littérature, on faisait lire des livres sans les placer dans l'Histoire et on faisait faire des rédactions. Je me rappelle qu'on avait des cours de «poésie», c'est-à-dire des moments bizarres où l'on nous demandait soudain: Voici un sonnet; maintenant vous m'écrirez un sonnet pour vendredi. Lors d'un de ces cours, j'ai donc écrit un sonnet. Dans les règles. Mais dans les règles, pour moi, voulait aussi dire que ce sonnet devait présenter quelque chose de différent, de bizarre. Juste pour le plaisir... C'est ainsi que je l'ai écrit, mais entièrement fait de verbes à l'infinitif présent. Ce sonnet, évidemment rejeté («trop facile, voyons...»), c'était tout à fait «normal», pour moi, de le faire ainsi...

Mais je crois que mes débuts tiennent peut-être du hasard. Il s'est agi que, dans la grisaille du quotidien d'un adolescent à Longueuil, j'aie vu apparaître des noms. Vanier, par exemple, qui faisait paraître son premier livre et qui faisait l'objet d'un article dans la Patrie ou dans Photo-Journal. Pas exactement le magazine littéraire par excellence, mais le premier qui ait parlé de ce jeune poète en termes élogieux. J'ai entendu parler de Péloquin de la même manière. Je ne connaissais personne qui écrivait, personne qui ait pu me diriger.

V. \& I. - C'est quand même une chose étonnante: entre 1962 et 1966, vous avez entre 12 et 16 ans, vous habitez Longueuil, ville de banlieue, et vous découvrez des choses qui sont loin d'être enseignées et même connues par l'ensemble de la population... et de la critique.

R. DES ROCHES - Le hasard, rien que le hasard. Si je me souviens, mes amis ne lisaient rien d'autre que des bandes dessinées. Ma famille était une famille québécoise tout ce qu'il y a de plus ordinaire, qui ne lisait pas, sauf les journaux, qui n'allait pas au cinéma, mais dévorait de la télévision. C'est 
vraiment un hasard. J'ai peine à croire à autre chose. Et s'il n'y avait pas eu ce hasard, mêlé à de la curiosité, ces articles en question m'auraient tout à fait échappé. Au contraire, je suis allé à Montréal, j'ai acheté Je de Denis Vanier. J'ai eu le goût. Pourquoi n'ai-je pas été rebuté par ce livre? Est-ce parce qu'à 10 ans, connaissant ce que faisaient Braque et Picasso, et Miro et Ernst, je trouvais déjà normal de déformer la réalité, la déformation étant plutôt une autre façon... normale de dire les choses. D'ailleurs, à cet âge, j'avais déjà acheté plusieurs monographiques qui faisaient partie d'une série publiée chez Marabout. Des peintres de toutes les époques, tous présentés de la même manière. Donc, quelque part, égaux, semblables, de même valeur, je ne sais pas... Peu importe les différences entre ces images, ces images avaient été peintes, donc elles avaient un sens, peu importe le sens, elles avaient un style, peu importe le style, elles étaient plutôt dues à une maîtrise, et c'était cette maîtrise qui m'importait. Le fait de pouvoir bien dessiner était, pour moi, plus important que ce que l'on dessinait. Le fait de pouvoir écrire ou de pouvoir bien écrire était plus important que ce que l'on écrivait. D'où le fait d'écrire des manifestes qui n'avaient strictement aucun sens; je croyais que seule était importante la maîtrise de ce style-là. Je pouvais donc copier sans crainte, disons m' «inspirer» copieusement: le premier recueil que j'ai écrit (en 1965) était fait d'une vingtaine de textes surréalistes «à la Vanier», complètement calqués, vers pour vers, poème pour poème.

Mais là n'est pas ce qui m'importe ou m'intrigue. Malgré les hasards qui ont fait que j'ai lu ce que j'ai lu, j'aurais bien pu en être horrifié, comme ce fut le cas de presque tout le monde. Moi pas. Je trouvais Vanier, Péloquin, Henri Vernes... normaux. Je lisais des livres différents en syntonisant différemment pour chaque livre. Mais ça n'explique pas tout et je ne sais pas comment expliquer ce côté de moi qui m'a tout le temps «impressionné»: disons que j'acceptais des choses inacceptables. J'acceptais des choses complètement à côté, sans que cela ne me cause quelque probleme. Je lisais vraiment n'importe quoi mais, en même temps, je lisais des vers complètement dérangeants pour certains, mauvais pour d'autres, modernes pour d'autres encore, et je me disais: bon, c'est tout simplement bien fait, c'est tout simplement drôle, ça fait naître des images, ça me donne le goût d'écrire.

Tout est venu très naturellement. Il n'était même pas question pour moi de me voir un jour ou l'autre dans la modernité. Du moins, pas vraiment ou pas clairement. D'ailleurs, c'est un mot que je ne connaissais même pas encore. Je n'ai rencontré ce concept qu'en lisant des biographies de peintres: je voyais bien que chez ces gens, il y avait un mouvement vers quelque chose de plus, vers quelque chose d'autre. On partait de l' «ancien» pour aller vers le «moderne». Mon image à moi de ce mouvement de l'ancien vers le moderne était très simple, voire simpliste: en peinture, on enlève de plus en plus de représentation - on déshabille les formes, et on ajoute de la couleur, des effets... En écriture... En écriture, on étonnait, on détonnait, on ne se justifiait pas. Pourquoi d'ailleurs se justifier puisque c'est naturel et que c'est ainsi que ça s'écrit. C'était et c'est presque encore la seule idée que je me fais de la modernité: on change, et cela peut aller autant vers l'avant que vers l'arrière. On change et seule l'idée de changer est importante: ne pas répéter, ne pas tomber dans le cliché, étonner si 
c'est encore possible... Quand je lisais mes petits livres sur l'art, il y avait Bosch et Manet, Giotto et Picasso. L'histoire de l'art aurait pu se passer tout au complet la même année! La seule chose que je voyais, c'est qu'on m'offrait, sans problème, plusieurs sortes d'images possibles. Magnifique. Pas de chicanes! Je ne voulais pas qu'une image disparaisse au profit d'une autre, je voulais plutôt que ce soit pluriel. Au départ, en écriture, j'ai à peu près fonctionné de la même manière, donnant à Françoise Sagan autant de bons points qu'à André Breton (et même quelques-uns de plus d'ailleurs!). Par la suite, j'ai appris à catégoriser...

Donc, une question de hasard, mêlée à de la curiositê mais aussi à de la paresse. J'étais curieux: je suis tombé sur Je. J'étais paresseux: je ne suis pas vraiment allé voir plus loin avant l'âge de vingt ans où j'ai commencé, presque par obligation, parce que je faisais maintenant partie d'un «milieu littéraire», à lire les livres de la modernité avouée.

V. \& I. - Denis Vanier habitait Longueuil. François Charron, de deux ans plus jeune que vous, habitait aussi Longueuil. Avez-vous voulu rencontrer Vanier?

R. DES ROCHES - Pas vraiment. À lire des auteurs américains ou français, je me faisais une idée de l'écrivain qui le plaçait dans un monde à part, un vedettariat spécial qui le rendait inaccessible au commun des mortels. Cette perception n'a rien de bien original. Même aujourd'hui, malgré tous ces écrivains que je connais de près ou de loin, la rencontre d'un «vrai de vrai» me donne encore la chair de poule. Je me souviens très bien de ma première rencontre avec Miron - il fut le premier véritable écrivain à qui j'aie parlé... et qui m'ait écouté. Même si je n'avais strictement rien lu de lui, je savais que je parlais à une légende. Je faisais face à quelqu'un d'opaque (dans le bon sens du mot), qui avait un passé, alors que moi, je n'en avais à peu près pas...

\section{V. \& I. - Mais Charron, lui, est apparu assez tôt dans votre carrière.}

R. DES ROCHES - Si je puis me permettre un cliché, ce fut mon premier compagnon de route. J'ai rencontré François Charron au secondaire, vers 1966. C'est là que l'amitié est née, et ça continue depuis, malgré les distances idéologiques d'un moment, les distances, le temps. Je ne me souviens pas des toutes premières années, mais j'imagine que ce furent les années des premiers échanges de livres, des premières discussions, des premiers essais de textes. Charron a toujours été plus passionné que moi pour tout ce qui touchait l'écriture. Ma sainte paresse! Il a lu ses classiques, lui. Nelligan, Saint-Denys Garneau, Rimbaud. Il est venu à l'écriture par la porte de la volonté, alors que j'y glissais d'une façon relativement passive. Intéressée, mais passive.

Mais c'est quand même ensemble qu'on a commencé à se prétendre écrivains. Et c'est dans le courant de l'êté 1968, un jour, qu'il m'a dit dans une lettre (Dieu sait pour quelle raison, on avait décidé de s'écrire plutôt que de se téléphoner, lui à Longueuil, moi à Montréal): $J$ ai découvert une revue qui s' appelle les Herbes rouges, $j$ ' ai envoyé des textes, pourquoi n'en enverrais-tu pas?. Un autre beau coup du hasard. J'ai publié dès le numéro 2 . 
La revue était et est toujours dirigée par François et Marcel Hébert. La correction des premiers textes s'est faite par correspondance, par téléphone. Ce n'est que plus tard, aux épreuves que je rencontrai les frères Hébert. Mais déjà, au cours de ces interminables échanges téléphoniques, $\mathrm{j}$ 'avais découvert leur passion pour la poésie, un autre type de passion, une passion pour ainsi dire désintéressée, puisque seul Marcel écrivait et qu'il était clair que jamais il n'en ferait une carrière. J'ai découvert leur rigueur, leur foncière honnêteté face au texte et à l'écrivain, leur approche no-nonsense de l'écriture littéraire. Rien n'était gratuit ou spontané. Lorsque mon premier recueil fut accepté, en 1969, par les Éditions du Jour, je rencontrai chez eux les frères Hébert et, pendant deux longues soirées, on travailla, un frère de chaque côté, chaque poème, chaque vers, vers par vers, mot par mot. De la dissection. À l'époque, je faisais énormément d'effets visuels, des effets d'échelle et de cascade, des mises en pages compliquées. Pendant ces deux soirées, j'ai subi ce qui me semblait la question. Chaque effet, s'il leur semblait suspect, devait être justifié. S'il était injustifiable, il sautait. J'ai appris tout le sens du mot «corriger». J'ai appris, de la manière la plus dure, que corriger n'est pas seulement changer quelque chose à un texte, mais bien rayer, effacer, faire disparaître. J'ai appris à me relire comme si ma vie en dépendait! J'ai aussi appris le sens du mot «cliché», que le cliché se cache derrière toutes les images et qu'on n'a plus besoin d'attendre cent ans avant qu'une expression devienne cliché. La modernité, à elle seule, est responsable de plus de clichés que toutes les autres littératures mises ensemble! J'ai donc rayé des vers du type les cuisses de la nuit, les insectes du désir. Ils me disaient: On n'écrit plus comme ça. Déjà? «Paul-Marie Lapointe a écrit cela en 48.» Ah, bon?... Et de me donner les exemples précis, les influences (provenant de livres que je n'avais pas encore lus, mais peu importe!), les do's and dont's. Enfin, après avoir charcuté des poèmes, mis des poèmes au panier, ils me proposaient des vers, des tournures différentes, ils me suggéraient des effets. Je me souviens, après la première soirée, avoir déclaré, défait: Ça n'a plus aucun sens! Ce n'est plus mon recueil! Ces corrections-là ne viennent pas de moi... je n'en suis pas l'auteur! Ce à quoi ils répondront spontanément par un rire général!

Ce fut, donc, un travail rigoureux et décourageant. Et la naissance d'une méthode de travail qui n'a à peu près pas varié depuis: chaque recueil soumis est lu et corrigé par eux — de moins en moins, bien sûr, au fur et à mesure que je continue à apprendre à écrire. Ce qui fut, au départ, une méthode plutôt intuitive, est devenu, par l'apprentissage du métier, une méthode plus sérieuse, plus réfléchie.

Mon lieu d'apprentissage aura été les Herbes rouges, avec tous les contacts faits dans cette espèce de lieu barbare, hors du temps: Huguette Gaulin, François Tourigny, Lucien Francœur, André Roy... Jusqu'à 18 ans, j'avais fait l'apprentissage de la poésie par le «vécu», si l'on veut. Mon bagage poétique s'était construit jusqu'alors, autant fantasmatique que lexical, au gré du hasard. Avec les frères Hébert, j'aurai appris le métier d'écrivain.

V. \& I. - De votre premier recueil refusé, en 1965, par Image et Verbe, jusqu'à votre premier poème accepté, en 1968, par les Herbes rouges, comment écriviez-vous? 
R. DES ROCHES - Sans méthode, de toutes les manières possibles, n'importe quoi, n'importe comment... Et ça ressemble plutôt à ma présente méthode d'écriture! (rires)

Comme pour la plupart des écrivains, j’ai commencé par écrire des poèmes ou des proses «poétiques», ainsi que je le disais plus tôt, inspirées de mes premières lectures, Rimbaud entre autres. Ce qu'en anglais on appelle des mood pieces. Toutefois, puisque je n'étais pas a priori un lecteur de poésie avisé, mais bien plutôt un lecteur de romans et de nouvelles de science-fiction, j'ai rapidement eu le goût d'écrire des romans et des nouvelles.

En 1964, pour mes 14 ans, j'ai eu ma première machine à écrire. Pour qui possède, comme c'était mon cas, une main d'écriture atroce, la machine à écrire est un don des dieux: elle permet une vitesse que je ne dépasserai, plusieurs années plus tard, qu'avec le micro-ordinateur.

La machine à écrire a provoqué chez moi une espèce d'hémorragie de textes, de débuts de textes, de bouts de textes. Leur simple «impression» sur la page donnait aux textes - si vous me permettez d'utiliser le terme - une opacité, une «vraisemblance» et une raison d'être particulière. Le geste d'écrire prenait même une tout autre valeur, accompagné du crépitement de la machine à écrire. Alors que le geste de peindre est large et évident, avec ses odeurs et ses rituels, le geste d'écrire à la main est mince, silencieux, détaché du résultat. La machine à écrire venait rajouter une dimension sonore, une dimension mythologique au geste d'écrire: je savais très bien, à 14 ans, qu'un écrivain sérieux (c'est-à-dire un écrivain américain) ne perdait pas de temps et vivait avec sa machine à écrire comme on vit avec une femme.

La même année, je commence à lire Châtelaine dont je découperai toutes les nouvelles pendant trois ou quatre ans. Il ne faut pas oublier que Châtelaine fut un des lieux privilégiés pour la nouvelle québécoise pendant des années. Jasmin, Thériault, des dizaines d'auteurs y sont passées. Lorsque Châtelaine cessa de publier des nouvelles, le genre est mort au Québec.

\section{V. \& I. - Pour renaître avec XYZ, entre autres.}

R. DES ROCHES - Exact. La nouvelle, comme le poème, est souvent un tremplin intéressant pour un jeune auteur, un endroit limité dans l'espace où apprendre à écrire. Le poème, toutefois, ne mène habituellement qu'au poème; il donne des habitudes qui sont incompatibles avec le roman, tel qu'on pourrait l'entendre lorsqu'on parle de texte volumineux, structuré, mettant en scène des personnages dont le comportement est plus ou moins calqué sur la réalité...

Enfin... Les premiers textes que $\mathrm{j}$ 'ai soumis furent deux nouvelles. Châtelaine, heureusement, me les refusa. Puis ce furent deux autres nouvelles écrites cette fois directement en anglais et envoyées à des revues de sciencefiction américaines. Refus assez évident.

C'est pendant ces premières années d'écriture que je développai mes fétiches d'écrivain. La machine à écrire (j'en ai possédé quatre, de plus en plus perfectionnées, avant d'opter pour l'ordinateur) donnait une «allure» à tous mes textes et une «allure» au geste même. $\grave{A}$ la main, pour retrouver quoi que ce soit de semblable, à défaut de me promener sur Saint-Denis avec des taches d'encre aux 
doigts, il me fallait de belles plumes, du beau papier, de beaux cartons, de beaux cartables, de belles bricoles... J'alternais: à la main, à la machine. Je commençais à la main, je continuais à la machine, puis à la main, je faisais des corrections, je redactylographiais, je revenais en arrière, je reprenais à l'infini, etc. Je mettais en pages, je me faisais mes propres éditions limitées (un exemplaire!)... je perdais le plus de temps possible. Quand j'ai découvert l'ordinateur, cela a été merveilleux, j'ai pu perdre mon temps d'une façon encore plus efficace!

Tout ceci pour dire que j'apprivoisais le geste d'écrire dans ses infinis mythes et ses infinies perversions.

V. \& I. - À un autre niveau, écriviez-vous avec (et à partir) des livres sur votre table?

R. DES ROCHES - J'ai toujours eu le goût de reproduire ce qui me plaisait, c'est-à-dire que je venais à une forme ou à un genre ou à un thème par une espèce de jalousie: C'est ce texte-là que j' aurais dû écrire... C'est ce texte-là que j'aimerais écrire... Au fur et à mesure que les rayons de ma bibliothèque se remplissaient, je me suis rapidement entouré de livres. Des livres autour du lit, des livres autour de la table de travail. Comme une collection de jouets en peluche... Ces livres $m$ 'habitaient, me stimulaient, et - plus souvent qu'autrement - me frustraient!

Certains développent des méthodes d'écriture rigoureuses, des heures de travail, des systèmes de références, etc. Mes méthodes ont toujours plutôt tenu du hasard. Du hasard et du mimétisme. Je lisais un texte et cela me donnait le goût d'écrire. Un texte semblable. Je lisais un poème, j'écrivais un poème. Je lisais un roman de science-fiction, je voulais écrire un roman de science-fiction. Je n'ai malheureusement jamais réussi à écrire un roman de science-fiction, ou un roman tout court. Le mimétisme pouvait aller jusqu'à essayer de reproduire, lorsqu'il m'en sautait aux yeux, des effets de style. Toute néfaste que puisse se révéler cette façon de fonctionner, c'est quand même là que j'ai appris le style: en regardant les phrases des autres, en essayant de voir comment c'était fait (sans analyser bien sûr, sans nommer les choses, sans me dire: $c^{\prime}$ est une inversion, c'est une métaphore, etc.), de voir qu'est-ce que cela fait naître, en essayant de sentir les choses. Je ne lisais pas à partir de la science, mais à partir d'une espèce d'intuition des choses. Intuition qui m'a sûrement fait manquer bien des choses, perdre du sens, mal comprendre beaucoup de bons et de mauvais textes. J'aime rarement revoir, en relisant un livre acheté à 17 ou 18 ans, le type de phrases soulignées à cette époque. Je me laissais si souvent prendre par des formules tordues que j'en ai honte!

Mais revenons à ce petit monde feutré que je me faisais avec mes livres tout autour... Ce que je voyais en écrivant, c'était des épines de livres, des couvertures. Ce que je voyais, c'était donc des mots au hasard, des titres - des expressions encapsulées, vivantes, pleines d'images (il faut se rappeler le genre de livres que je lisais!) -, mais aussi, sur les murs, des photos, des affiches. J'ai toujours couvert mes murs, l'espace spécifique en face de ma table de travail, de photographies dont le ton résumait ce que je croyais être, ce que je croyais vouloir dire, ce qui m'obsédait. Une bonne part des premiers poèmes, des poèmes 
de Corps accessoires (recueil écrit en 1968) et de certains poèmes de l'Enfance d'yeux (écrit en 1969-1970), sont truffés d'expressions, de mots, de phrases entières, pigées à même ces couvertures, ces images. Certains passages de Reliefs de l'Arsenal (1974) sont des descriptions relativement minutieuses de photographies découpées dans des revues américaines et françaises. «Theda Bara», qu'on retrouve dans Corps accessoires: c'était à partir d'une grande affiche de cette actrice tenant le rôle de Cléopâtre. Je ne l'ai jamais vue au cinéma, mais peu importe. L’image était hypnotique. Je glanais, donc, dans ce qui était là, dans ce qui poussait là, parce que mes murs se couvraient parfois jusqu'au plafond de ces images-décor. Je glanais, simplement, dans le «matériau» à ma disposition, sans vouloir caviarder, sans vouloir voler ou plagier, mais pour l'effet. L'effet me semblait intéressant, valable, de la matière à considérer comme toute autre matière. Chose bizarre encore, comme je l'expliquais plus tôt, je n'ai jamais senti de problèmes face à ces emprunts et à quelque effet de style que j'aie voulu produire. C'était «normal» d'écrire ainsi, d'écrire, par exemple, seins Laura Secord centres mous à 18 ans. J'y voyais, évidemment, quelque chose qui devait être de la modernité, mais sans plus. Quels mécanismes?... Je n'aurais pas pu écrire autrement. Je n'ai jamais pu écrire autrement de la fiction.

V. \& I. - Un peu de cuisine, Roger Des Roches. D'où proviendrait ce vers en particulier?

R. DES ROCHES - Ce vers-là vient probablement d'une boîte qui traînait sur ma table. De mon goût immodéré pour le chocolat... de ma délicieuse obsession pour les seins de toutes les femmes... et aussi du fait que je préférais les chocolats aux centres mous aux chocolats aux centres durs. Une image comme ça, aussi simple qu'elle puisse paraître - mais je sais qu'elle ne l'est pas nécessairement - m'amusait au plus haut point car, avant, pendant ou après, j'y avais caché un petit monde à moi, j'y avais inséré des jeux d'initié, tout en reconnaissant que j'étais ce seul initié et que l'image devait vivre d'ellemême, en surface s'il le fallait. Il y a ainsi plusieurs autres vers ou phrases que certaines personnes «comprennent», mais que les lecteurs dits «normaux» ou «passants» ne pourront jamais «comprendre».

V. \& I. - Le titre de votre premier recueil (Corps accessoires) est certainement très important puisqu'il deviendra, avec une légère modification («Tous, corps accessoires...»), le titre de la rétrospective de la première partie (1968-1973) de votre œuvre. Que voyiez-vous, à l'époque, dans ces mots (un nom et un adjectif, ou deux noms), dans ces notions de «corps» et d' «accessoire»?

R. DES ROCHES - Corps accessoires n'était pas le titre original de ce premier recueil accepté par les Éditions du Jour. Le titre était les Doux Émétiques. Heureusement, les frères Hébert l'ont trouvé idiot (On n'écrit plus comme ça!...). Corps accessoires, si ma mémoire est bonne, a été proposé par l'imprimeur Ginette Nault. Il provient d'un poème du recueil suivant, l'En- 


\section{fance d'yeux.}

Ceci dit, je ne crois pas avoir eu, à l'époque, une idée très claire de ces notions. Sans parler de «spontanéisme», mon écriture tenait quand même beaucoup des effets de choc. Mais le corps, c'était toute la sexualité bourrée dans un seul mot, une sexualité refoulée, non vécue ou à peine vécue alors. Étant un late starter, j'avais plutôt des connaissances disons... littéraires de ce qu'était le corps des autres, le corps de l'Autre. Le corps de Corps accessoires, c'était tout ce qui m'était «interdit»: tout ce que je n'avais pas touché, tout ce que j'imaginais, tout ce que j'avais vu, lu et pressenti dans la littérature érotique, dans la photographie érotique, dans la peinture érotique. C'était tout ce que je devinais de ce que devait être un corps: j'ai donc écrit le mot «sein» avant de jamais toucher un sein! Tout n'était que fantasme, imagination, interdits personnels, espoirs exacerbés. Un jour, je goûterai à ses seins. Un jour je saurai qu'est-ce qu'il y a dans un sein pour que je pressente déjà que ça résume toute la douceur du monde. Un jour, ça deviendra réel. S'il existait un quelconque intérêt à le faire, on pourrait fort probablement lire une différence entre Corps accessoires et la fin d'Interstice (écrit en 1971) quant à la façon de nommer, d'épithétiser, de sentiŕ le corps, différence due à un début de sexualité, sexualité évidemment très restreinte à l'époque. Une chose toutefois était sûre: je ne m'étais pas trompé sur l'incroyable folie de douceur que représentent les seins d'une femme.

V. \& I. - Le dernier vers d'Interstice (air qui suit chair) peut donc aussi s'entendre «R. qui suis chair».

\section{R. DES ROCHES - Ou «R. qui suit chair»... je l'ai à l'œil!}

Et accessoires... Parce que, justement, on parle de fantasme, d'appropriations faciles. On parle de corps qui s'échappent. S'ils s'échappent, c'est qu'ils ont cours. La littérature érotique m'offrait des corps disponibles: des façons de m'en tirer, des corps relativement silencieux, mais surtout en accord avec ce que j'étais: disponible et transparent. Je ne voyais pas en la littérature érotique (et, ceci dit, je ne vois toujours pas aujourd'hui en la pornographie) une façon de réduire l'autre au plus petit commun dénominateur. Je voyais bêtement une façon de jouir gratis, et de jouir bonnement, et de jouir sans comptabilité. Il y avait la disponibilité, l'excès - prudent, mais l'excès quand même. Un bonheur de corps que bien souvent la «vie réelle» ne permet pas. Peu importe. Tous corps accessoires...

V. \& I. - Quand vous prononcez ces mots, vous faites de «tous» un pronom, ce qui implique une pause entre «tous» et «corps». Pause marquée, d'ailleurs, par une virgule dans le titre de la rétrospective.

R. DES ROCHES - Si l'on regarde le poème dont ce vers est l'envoi, on voit qu'il y a dans les trois premiers vers une volonté d'énumérer un certain nombre d'états ou de choses pour arriver à une sorte de conclusion: «voici, ce sont tous des corps accessoires». 
V. \& I. - Ce sont aussi des corps auxquels on voudrait avoir accès, des corps qui permettent l'accès.

R. DES ROCHES - Comme je 1'ai dit déjà, le sens n'était sûrement pas voulu aussi fin, mais il est clair que la notion de... prothèses, comme choses qu'on accroche ou qu'on décroche, se cache quelque part derrière. Mais le corps de l'autre est inéluctablement une chose, une chose qui est, peu importe les prétentions de «fusion», externe à soi. «L'autre, c'est l'autre; voici moi, voici l'autre» ai-je écrit récemment. Chose qu'on ne peut vraiment s'approprier, peu importe la douceur ou la violence du toucher, mais qu'on peut toucher, repousser, reprendre, oublier... Accessoires, donc, au sens où les choses ne sont pas soi, mais autres. D'ailleurs, cette distance astronomique, je ne la comprendrai - ou n'y mettrai un nom - que plus tard, quand je citerai l'Érotisme de Bataille (dans SPACE-OPERA (sur-exposition), écrit en 1972-1973) qui dit: Entre un être et un autre, il y a un abîme, il y a une discontinuité. Entre deux êtres qui se parlent, il y a un abîme de corps (tu n'es pas mon corps, comme se dit l'enfant qui, n'ayant pas encore appris son corps, voit son pied comme un objet étranger), un empêchement. Adulte, on met une frontière, de la tête aux pieds, à ce qu'est notre corps, ceci incluant le dos qu'on ne voit jamais, incluant les zones grises et les points aveugles. L'autre corps, peu importe combien collé, combien intime en paroles et en gestes il est, quelque soit la pénétration, il reste qu'il ne fait pas partie de soi. Si jamais vous aviez l'intention de me le demander, voilà peut-être le grand thème de toute mon «œuvre»: l'autre, c'est l'autre; je veux moi, je veux l'autre...

V. \& I. - Qu'est-ce que c'était, alors, il y a presque vingt ans maintenant, l'écriture, au sens où vous pouvez actuellement l'entendre et au sens où on l'entend aujourd'hui en théorie, au Québec, avec des revues comme la Barre du jour et la Nouvelle Barre du jour, comme les Herbes rouges?

R. DES ROCHES - Mettons immédiatement une chose au clair. Je ne me vois pas - je ne me suis jamais vu - comme un théoricien ou comme un intellectuel. Mes connaissances ont toujours été limitées au niveau théorique, et j'ai même toujours eu une sainte horreur de la théorie, de quelque couleur qu'elle soit, de gauche, de droite, de la modernité ou de la non-modernité. Ayant fondé Stratégie avec François Charron en 1972, j'ai même dû quitter après le quatrième numéro: je ne comprenais même plus les articles que nous publiions ou, pire encore, je n'en voyais non seulement pas la pertinence, mais je commençais à croire (et je le crois encore aujourd'hui) que ce type de thérie venait étouffer le travail d'écriture. Ce fut une des raisons (les autres furent «politiques») qui amenèrent un jour une espèce de brouille entre Charron et moi. Heureusement, on vieillit tous, les brouilles disparaissent, et certaines valeurs, si elles peuvent demeurer ou se transformer avec honnêteté, certaines valeurs, donc, ne prennent plus la même importance dans la vie de tous les jours. On salue aujourd'hui, dans la rue, des gens qui ne partagent pas les mêmes opinions que soi. Business is business. Tout ceci pour dire que mon discours théorique, mon vocabulaire théorique, se révèle très limité: j'absorbe et j'《intuitionne». Au 
niveau théorique, je demeure un silent partner de la poésie. J'évite donc, chaque fois que je le puis, de m'enfoncer dans la verbalisation d'une quelconque théorie de l'écriture. Je demeure sur mes gardes et je préfêre dire: Lisez mes textes. Comprenez ce que vous pouvez. Théorisez à ma place. On verra toujours!

Si l'on remonte à la préhistoire, entre 1962 et 1965 , pour moi, le mot «écriture» n'existait pas. Le mot «écrivain» existait, mais relégué dans une espèce de no-man's land ou de panthéon flou d'où tout pouvait sortir. Les mots «peinture» et «peintre», cependant, existaient. C'est-à-dire que j'en avais une image relativement plus précise, quoiqu'elle était assez naïve par moments. Le mot «écriture» n'existait pas, mais déjà le geste d'écrire avait pris suffisamment d'importance pour m'indiquer, à voix basse, que j'étais en train de devenir écrivain. Certains jours, toutefois, devenir écrivain, ça voulait simplement dire devenir célèbre. Le livre était un objet à part de l'écriture. L'écriture se limitait souvent à la personne de l'écrivain, donc à sa notoriété ou à ses excentricités.

L'écriture, je l'ai comprise, je l'ai mal comprise; je l'ai mieux et mal comprise plus tard. C'est devenu un métier, peu à peu, mais c'est toujours, d'abord et avant tout, entre 62 et 65 , un geste. C'est le corps qui exulte sur un espace restreint. Entre 1968 (mes premiers poèmes publiés) et 1970 (mon premier livre publié), c'est devenu, sans que je $m$ 'en aperçoive, une raison de vivre, ou une façon de vivre. Avec déjà, malgré tout le discours moderne et non moderne, un grain de no-nonsense: plutôt que devenir une recherche de l'absolu ou je ne sais quoi, l'écriture a toujours été pour moi: J'écris parce que j'ai le goût de produire, parce qu' il faut produire, et je produis pour publier. Il n'est pas question d'écrire un texte dans «le vide», pour mes tiroirs, un texte qui ne soit pas destiné, d'une façon ou d'une autre, à être publié. S'il ne l'est pas, c'est qu'il n'est pas publiable. Á mes yeux ou aux yeux de mes éditeurs.

\section{V. \& I. - Vous acceptez la critique? À ce point?}

R. DES ROCHES - De certaines personnes. Écrivains ou pas. Ma réaction de panique face au travail de correction et de révision sur mon premier livre ne se branchait pas sur un refus de la critique, mais sur ma vision relativement naïve de ce qui est «personnel» et de ce qui ne l'est pas. Je ne croyais pas qu'une personne puisse «travailler» mon texte à ma place. Une question de propriété privée mal placée. Mais je savais qu'on pouvait en savoir plus que j'en savais. Je ne savais pas comment cette expérience de mes pairs pouvait se jouer à l'intérieur de mes propres textes.

D'ailleurs, j'ai même eu un jour un petit recueil de poèmes, près de 15 ou 20 poèmes tout frais, tout beaux, de refusé par les frères Hébert en 1971 , je crois. Ils m'ont fait comprendre: On n'écrit pas comme ça... C'étaient tous des poèmes qui se réduisaient à des effets sonores plus ou moins baroques. Au profit des exégètes, je pourrais dire que $j$ 'en ai repris certains vers dans une courte «nouvelle» intitulée la princesse radiophonique (publiée dans Autour de Françoise Sagan indélébile, puis dans Tous, corps accessoires...). Bon gré, mal gré, j'ai dû accepter la critique.

V. \& I. - Beaucoup de vos premiers poèmes jouaient sur les effets sonores... 
R. DES ROCHES - J'ai eu ma période «sonore» dans plusieurs textes de l'Enfance d'yeux, d'Interstice, dans plusieurs textes, tout compte fait, produits autour des années 70-73. Plus tard, les effets sonores reviendront, mais "cachés» cette fois, dans des rimes intérieures, des effets d'allitérations, etc. Le problème majeur des effets sonores dans la poésie, c'est qu'ils vidangent le sens avec une infinie facilité! Même lorsqu'on se prétend surréaliste, quelque part il y a sens - les images font sens. Dans certains des poèmes d'Interstice, justement à cause de ces effets de son, j'en arrive, à la relecture, plusieurs années plus tard, à avoir de la difficulté à $m$ 'y retrouver...

Pour en revenir à ma façon de voir l'écriture, on pourrait dire que j'ai eu, à un moment donné, autant de concepts de ce qu'était l'écriture que j'avais écrit de livres. J'ai même cru qu'écrire était une espèce d'état d'âme, un état second, peutêtre même une «mission» (voir aussi, plus tard, vers 1975-1977, ma vague période marxiste). Mais malgré toutes ces explorations dans ce qui est ou ce qui n'est pas l'écriture, c'est le goût d'écrire qui l'emportait, de toute façon, sur toute raison d'écrire. J'écrivais par plaisir, par plaisir pervers, par retour du plaisir que les autres avaient à lire mes textes. Plus j'avançais en expérience, plus j'atteignais une certaine maîtrise de mon écriture, plus le plaisir prenait de l'importance. Le plaisir d'écrire à jamais associé au plaisir de publier. C'est donc dire qu'une année sans livre(s) publié(s) était - et est encore une année désastreuse. Récemment, j'ai eu quelques années désastreuses. Jamais écrire n'a été aussi difficile!...

Le plaisir... De plus, je valorise l'écriture, le geste, les pratiques et la recherche et la rigueur, jusqu'à parler de «métier»: je pratique le métier d'écrivain, j'ai donc des responsabilités vis-à-vis l'écriture, les écrivains, les lecteurs. Responsabilités peut-être minces, bien entendu, mais qui tiennent du professionnalisme, de la façon de croire à ce que l'on fait, de toujours vouloir mieux le faire, de ne pas rechercher les solutions trop faciles, d'en connaître plus, etc. Ma notion de métier vient tout droit du métier de typographe que $\mathrm{j}$ 'ai exercé pendant près de 10 ans. Le métier de typographe est extrêmement codé. Le typographe passe par un apprentissage, par un compagnonnage, et l'écriture va toujours passer, pour moi, d'un apprentissage à un compagnonnage, d'un apprentissage à une maîtrise. Quelle sorte de maîtrise? Je ne sais pas vraiment. Si l'on se dit moderne, qu'entend-on par maîtrise, alors que cette maîtrise-là était justement ce que les «anciens» prônaient lorsqu'il s'agissait de cataloguer les «bons» textes et les «mauvais» textes. Peut-être que le type de maîtrise dont je parle - dont je sens l'importance sans pouvoir véritablement la nommer - tient de plusieurs qualités mouvantes, changeantes selon les époques, qui s'additionnent en quelque sorte - qu'on peut reconnaître a posteriori seulement. Je ne sais pas...

J'en reviens toujours à ma difficulté à analyser, à «théoriser»... Heureusement, je n'ai presque jamais tenté de théoriser l'écriture - sauf en de rares exceptions comme dans le Corps certain (écrit en 1974-1975), la Promenade du spécialiste (écrit en 1976-1977) et quelques autres textes relativement plaqués. Lorsque je ne me suis plus senti le besoin de théoriser, j'ai plutôt essayé de décrire de la façon la plus belle possible le geste d'écrire, l'obligation d'écrire. Cette obligation qui produit en moi un sentiment d'impuissance très proche de la frustration sexuelle, lorsque je n'arrive pas à produire au rythme 
où je le veux, de la manière dont je le veux, avec les bons mots et les bonnes images...

V. \& I. - En regardant un certain nombre de vos manuscrits, je constate que vous écrivez autant en vers qu'en prose, ce qu'on peut sans difficulté apprécier à la lecture de vos livres, surtout vos plus récents. Je constate également, ce qui est plus curieux, que vous avez, certaines fois, réécrit en vers un texte en prose ou l'inverse, comme si vous hésitiez sur sa forme extérieure.

R. DES ROCHES - Voici venu le moment où l'on aborde mes plus grands fétiches... après la chair des autres.

J'ai toujours hésité entre la prose, associée pour moi au roman, au roman sérieux, au romancier, à l'écrivain sérieux, celui qui produit des volumes épais, de vrais livres, et la poésie, les vers, les efforts à courte portée, le poète, l'écrivain à petits livres, qui fait dans l'absolu. Je rêve encore, aujourd'hui, d'écrire un roman (un roman du main stream, compréhensible à tous les niveaux), je rêve encore d'écrire un vrai livre, comme si le roman était une forme hiérarchiquement plus élevée que la poésie: on en dit plus, on est lu par plus de personnes, l'effet de performance est plus grand. On écrit de la poésie lorsqu'on ne peut pas faire autrement!

Je me dis ça habituellement dans mes périodes de fatigue, de manque de confiance ou d'idées. Dieu sait que j'ai essayé plus d'une fois d'écrire un roman pour enfants, pour adolescents, un roman de science-fiction ou d'horreur pour adultes, des nouvelles, des contes... et je sais que peu importe le résultat passé, je vais toujours essayer à nouveau. Cette espèce de rêve de performance me poursuit encore. J'hésite constamment entre la prose et la poésie. Lorsque j'ai une bonne idée, je voudrais immédiatement la mettre en prose - comme pour produire peut-être quelque chose de plus important. Par la suite, lorsque je suis enfin rassis, rassasié ou en meilleure connaissance de cause, je reviens à la poésie, puisque c'est ma façon d'écrire - d'écrire le mieux. C'en est presque ridicule. Je commence, disons, en prose. Une idée. Déjà, je vois le livre, je pourrais même en décrire la couverture (et pourtant, je n'en ai pas écrit plus de dix lignes!)... Très rapidement, ça bloque. Impossible d'aller plus loin. C'est quoi la «description»? Comment on fait pour croire à un personnage?... Si le «sujet», si le thème tient debout, je m'essaie en vers. Si je bloque à nouveau, sur la forme poétique cette fois, croyant me répéter, croyant tomber dans le cliché, je me dis: Ça irait sûrement mieux si j'étais en prose. J'essaie en roman traditionnel, ça bloque. J'essaie en roman moins traditionnel, ça bloque encore, mais pour d'autres raisons. Au bout d'un certain temps, je reviens enfin en vers et le livre s'achève, portant les marques - que moi seul je pourrai déceler - de tous ces essais infructueux. Le meilleur exemple que je puisse donner de ce jeu de pendule fantasmatique, c'est mon prochain livre: Tout est normal, tout est terminé (écrit en plusieurs temps, de 1985 à 1987, incorporant même, si je ne m'abuse, des extraits de textes écrits en 83!). Ce livre tient dans 22 pages manuscrites. Le travail préparatoire, oscillant constamment de la prose au poème, fait près de mille pages! Ce livre, avec ses matériaux tirés de plusieurs blocs de prose et de poésies non publiés, agencés par ajouts et par retraits, a fini 
par devenir une structure cohérente, une véritable architecture. Écrire ainsi, par bouts et par vols de moi-même, $m$ 'a causé de véritables problèmes de conscience: Je n'écris plus rien de neuf! C'est mon dernier recueil! Bien sûr, puisque j'ai vu et revu les mêmes textes, dans leurs dizaines de versions, tout au long de ces trois ou quatre années. J'ai été le seul à les voir, pourtant, ces versions! Mais j'ai fini par m'apercevoir - sans toutefois m'en convaincre tout à fait, je dois l'avouer - qu'un recueil peut être une réussite issue d'une suite d'échecs, une réussite par laquelle le poème peut renaître plus tassé, plus brillant, parfait (comme un diamant avec ses facettes est parfait... en voilà un beau cliché!). À la suite de toutes ces transformations, de la chenille au papillon (en passant par le dinosaure!), le recueil s'est imposé à un point tel que je ne peux rien ajouter, pas même une virgule. Le recueil se referme sur lui-même.

V. \& I. - Nous avons parlé des influences des origines. Quelles sont les lignes principales de ce que vous avez lu et écouté dans les années 1970 et 1980 ?

R. DES ROCHES - Commençons par l'oreille... De 1963 à aujourd'hui, j'ai toujours écouté la même chose: du rock, du rock et toujours du rock. Du rock figé entre 63 et 75 . Figé parce que j'ai peu de connaissances de ce qu'est la musique d' «aujourd'hui». Pour ne pas effrayer mes lecteurs, disons que j'apprécie quand même un peu le classique, mais, soyons honnête, ce qui me plaît dans le classique, c'est tout ce qui ronfle, tout ce qui tonne. J'ai une oreille pompier.

Même si cela paraît moins actuellement, le rock a toujours accompagné mon écriture: qu'on lise Corps accessoires ou qu'on lise l'Imagination laïque, on retrouve plusieurs vers en anglais, des rythmes d'écriture qui s'approchent du rythme du rock, des effets de répétition, certains types d'ellipses, de colorations...

Côté lectures, je dois avouer - à ma relativement petite honte - que j'ai presque abandonné la lecture de la poésie. Ou est-ce la poésie qui m'a abandonné? Je me découvre moins de ferveur qu'à l'époque de mes premiers livres. J'attends les découvertes, tout en lisant quand même certains auteurs du Québec: Charron, Roy, de Bellefeuille, Labine.

Parallèlement au rock, je lisais de la science-fiction. Aujourd'hui, j'opte pour l'horreur, le surnaturel, l'épouvante: Stephen King, Peter Straub, Ramsay Campbell et compagnie. J'ai découvert dans ces textes parfois très rococo, parfois très réactionnaires, un imaginaire particulier, frémissant, hautement sexualisé. De plus, l'horreur repose sur une certaine sorte d'émerveillement, d'étonnement dévié. J'aime qu'on me surprenne, de toutes les manières...

Comme si j'étais vieux... La poésie moderne ne me surprend plus. Les quelques auteurs qui m'intéressent poursuivent leur œuvre qui s'est détachée de toutes les expériences du passé, du proche passé. La relève travaille à un niveau qui m'est étranger. Un travail trop universitaire chez certains, un travail trop différent chez d'autres. Peut-être est-ce normal - dramatique mais normal - que cette exclusion de ce qui vient après. J'ai beau me convaincre en me disant que beaucoup d'auteurs de la relève fonctionnent comme s'il s'agissait de produire des travaux universitaires, ça ne peut pas tout expliquer et ça ne peut surtout pas s'appliquer à tout le monde... On verra... 
Aujourd'hui, donc, j'opte pour une certaine forme de facilité - de la musique facile, des films faciles, des livres faciles. Mais aussi de très beaux livres auxquels je n'aurais accordé aucune importance à l'époque des grands refus: Milan Kundera, Raphä̈lle Billetdoux, Umberto Eco... Je me contente de lire ce qui me pousse à écrire, d'où que ça provienne...

\section{V. \& I. - Sentirait-on peut-être aussi un certain découragement?}

R. DES ROCHES - Lorsqu'on connaît les chiffres de vente des livres québécois, il y a de quoi se décourager, auteurs comme éditeurs. Les tirages ont diminué de 1000 à 800 , de 800 à 600 , de 600 à 500 . Les livres disparaissent des librairies au bout de trois semaines, sont mal distribués, sont boudés par la critique... Malgré les différents prix remportés par les membres de notre génération, on assiste à un phénomène invraisemblable: jamais dans toute l'histoire de la «modernité» québécoise n'a-t-on été aussi mal connus, mal lus, mal enseignés. Au moment même où certaines formes excessives se tassent, où le travail de recherche s'est décanté et commence à donner des œuvres de maturité. Nous commençons à disparaître, à n'être connus que de nous-mêmes comme nous n'étions connus que de nous-mêmes en 1970 dans la cuisine des frères Hébert. C'est le retour au no man's land et au no woman's land.

V. \& I. - Après la rétrospective intitulée Tous, corps accessoires... qui rassemble les textes écrits entre 1968 et1973 - ce qui constitue de ce fait la première partie de votre œuvre, quelles autres divisions faites-vous?

R. DES ROCHES - En fait, SPACE-OPERA, dernier recueil de la rétrospective, étant le «début» de Reliefs de l'Arsenal (question de forme, de thème, d'utilisation de la science-fiction), ce récit aurait dû s'y ajouter et boucler la boucle.

La seconde partie de l'œuvre pourrait fort bien aller de la Publicité discrète (écrit en 1974) à la Promenade du spécialiste (terminé en 1977). Le texte en prose qui clôt ce dernier recueil, «Épisodes \& le risque», j'en fais un cas à part et je le joins plutôt au cycle qui démarre avec les Lèvres de n'importe qui (écrit en 1977-1978) et se poursuit aujourd'hui. «Épisodes \& le risque», c'est un style particulier de prose sur lequel je vais longtemps travailler et qui va finir par donner ces blocs de prose relativement à la mode au début des années 80, ainsi qu'un style dialogué - qu'on retrouve dans Pourvu que ça ait mon nom (écrit en 1978-1979, en collaboration avec Normand de Bellefeuille) - grâce auquel je parle presque exclusivement du couple. Bien sûr, j'avais déjà parlé du couple, dans la Vie de couple par exemple (écrit en 1975-1976), mais c'était emmêlé de prétentions psychanalytiques, philosophiques ou politiques. Je dis bien "prétentions» parce que ces notions, je ne les sentais que comme défenses face à l'élite intellectuelle de l'époque: un texte n'est pas un texte s'il ne parle pas du père (en le rejetant, évidemment), un texte n'est pas un texte s'il ne défend pas les femmes coûte que coûte (comme si les femmes n'étaient pas parfaitement capables de se défendre elles-mêmes). Ces notions-là, je ne les possédais pas plus qu'il ne faut. Oublions ça!... 
La première partie (68-74) se tient assez bien: c'est l'exploration langagière, les effets de style et, au sens large, les jeux de mots; c'est aussi l'apprentissage de la poésie et de la prose. Dans la deuxième partie (75-77), je parle un peu plus du couple, mais c'est emmêlé à d'autres effets d'écriture, plus syntaxiques que lexicaux et sémantiques. La troisième partie, c'est le résultat d'un décantage: $\mathrm{j}$ 'abandonne les effets psychanalytiques, les effets politiques (moralisateurs) pour en arriver à ce qui m'apparaît le problème (mon problème, notre problème?): le corps à corps, l'être face à l'autre et l'abîme qui les sépare. La troisième partie est vraiment l'«analyse» émotive, presque lyrique, du couple, du couple éphémère ou pas, plutôt éphémère que pas, ou du non-couple, et de la rupture du couple et de ce qui s'en suit, la solitude.

Le dernier recueil (Tout est normal, tout est terminé) est ce que je pourrais appeler ma «maturité» d'écrivain, bien qu'on en soit toujours à l'atteindre cette maturité: avoir moins honte de telle phrase, mieux contrôler ce qu'on veut dire, «cristalliser» plus souvent de beaux vers, de belles phrases.

V. \& I. - De la première partie à la troisième partie, il y a aussi de plus en plus de brouillons.

R. DES ROCHES - Un écrivain qui écrit, c'est une usine à brouillons. Faudrait songer au recyclage!

V. \& I. - Au début, on lit presque uniquement des vers sans ponctuation, assez abrupts. Ensuite, avec la ponctuation apparaît la phrase dans toutes ses composantes, mais les sauts de toutes sortes (dans la Vie de couple particulièrement) demeurent très nombreux. C'est dans les textes de la deuxième partie que vous utilisez à peu près tout l'appareil éditorial: les notes, les mots et les locutions inachevés, les guillemets, les parenthèses ouvertes et non fermées (par oubli peut-être)...

R. DES ROCHES - Par oubli très souvent! La modernité a de ces petits secrets...

V. \& I. - ...une ponctuation foisonnante, le romain et l'italique, etc. La troisième partie est généralement assez «claire»: énoncés aphoristiques, ponctuation sage.

R. DES ROCHES - Sage en apparence seulement. Et d'ailleurs, rappelezvous que l'Imagination laïque ne présente aucune ponctuation. Mais toutes ces expériences ont fait leur œuvre. Je crois très bien comprendre actuellement ce qu'est la ponctuation, l'ayant utilisée à tort et à travers mais aussi en connaissance de cause, faisant exprès pour faire subir aux phrases des torsions impossibles: en torturant la ponctuation, j'en suis peut-être arrivé à vraiment sentir et faire sentir la marque du souffle qu'est la ponctuation. Chaque époque possède son souffle particulier, les phrases sont «soufflées» différemment. L'expérience ultime - pour ce qui me concerne -, c'est près de 1500 vers répartis dans six sections, six «souffles» d'ailleurs, où, paradoxalement, il n'y a pas de 
ponctuation...

J'en arrive maintenant à des phrases qui, ponctuées, le sont, en bonne majorité, correctement. En bonne majorité et en apparence. Lorsqu'on gratte la surface, on trouve des surprises.

V. \& I. - C'est dans le Corps certain (écrit en 1974-1975) que je lis: virgule dis-je est petit moteur vers l'arrière...

\section{R. DES ROCHES - Qu'ai-je bien voulu dire?... (rires)}

La virgule, graphiquement, est une petite boule avec une queue qui pointe vers l'arrière... C'est la marque de l'incise, de l'hésitation, du souvenir. Et si l'on revient trois pages avant, donc vers l'arrière, on peut lire: elle est aussi très mobile cette visite des virgules - on la verrait facilement changer tout notre corps s'il est écrit que nous avons des attributs précis, il l'est écrit. Comme si un agent extérieur venait changer le sens voulu au départ du texte, du corps du texte qui est plus près de notre corps que notre corps l'est du corps de l'autre. Comme la virgule change le texte, elle change mon corps. Il y a ces signes du souffle que j'ai mis dans le texte, signes du coma - et virgule, en anglais, c'est: comma -, donc signes d'une espèce de disparition de l'écrivain en arrière, de la neutralité, et il y a l'effet de mur que pose le texte face à la lecture (on n'entend, ne comprend plus rien, comme dans le coma). La virgule mettant tout le temps des temps d'arrêt où l'on peut réfléchir à ce qui vient d'avoir lieu, elle est donc un moteur qui pousse la compréhension vers l'arrière. J'ajouterai que c'est dans le Plaisir du texte de Roland Barthes que j'ai trouvé le titre du recueil: le Corps certain. Ici, encore, jouent le texte et le corps. Et la virgule comme doigt ou sexe, petit sexe.

V. \& I. - Le dernier titre (Tout est normal, tout est terminé) est un titre remarquable pour plusieurs raisons, dont la moindre n'est pas celle qu'on appelle la «double contrainte»: de chaque côté d'une virgule, on est pris. Ce qui est normal est terminé, ce qui est terminé est normal.

R. DES ROCHES - Déjà, dans la Promenade du spécialiste, il y a quelques titres de textes ainsi construits: «Le mot «choix», le mot «désespoir» dans ses exercices» ou encore «Tendresse, irritation; la peau juste entre les deux». Quant au dernier titre comme tel, il vient d'un vers du texte, ou d'une des versions en prose le précédant, intitulée le Célibataire. Dès que $j$ 'ai «vu» ce titre, il s'est imposé par son effet. Tout est normal: je continue comme si de rien n'était, mais tout est terminé: je parle de la fin d'un couple (dans ma vie privée, très précisément), je parle presque de mon désir d'en finir avec la poésie (non «après moi, il n'y aura plus de poésie», mais «d'après moi, je ne ferai plus de poésie»); pourtant ma vie privée se résume souvent à la recherche du couple - dans son image la plus conventionnelle même; et pourtant je ne puis m'empêcher de vouloir que certains mots me ressemblent - et ils me ressemblent le plus quand je le fais en poésie. Comme si tout ce qui est normal devait être terminé, recherché désespérément et abandonné.

Côté «double contrainte», on peut en trouver une facile dans Poème, 
attention!: Poème, le côté «êthéré», le désordre apparent, attention!, le rappel à l'ordre.

Tous mes titres s'imposent par une image. La Promenade du spécialiste, c'est faussement XVIIIe, c'est franchement ironique. Le Corps certain, c'est peut-être l'effet le plus théorique que j'ai pu donner à un titre, mais où ce titre m'a séduit, c'est par son aspect surréaliste. L'Imagination laique se veut pamphlétaire: arrêtons de penser à Dieu sait quoi, à l'inspiration, à la poésie qui n'est pas de la poésie, mais de la fiction ou de la prose ou Dieu sait quoi, et faisons de l'écriture une imagination chargée, une imagination de métier. L'observatoire romanesque m'est venu dans un... éclair, c'est bien sûr un jeu de mots sur... l'Osservatore romano. Sans oublier le sous-titre de «confidences», boutade de Normand de Bellefeuille si je me souviens bien, ajouté à Pourvu que ça ait mon nom. Boutade, oui et non. Ce sont, maquillées même pour nous qui les écrivions, des confidences hors de l'oreiller, à propos de l'oreiller, à propos d'amitiés et de recoupements d'amitiés de femmes, à propos de rapports amoureux desquels nous - Normand et moi - étions témoins, en connaissance ou en méconnaissance de cause.

V. \& I. - Je regarde la photographie de la quatrième de couverture: vous êtes chacun dans une boîte téléphonique, séparés par une autre boîte téléphonique, occupée elle aussi - mais par un passant (qui, lui, parle au téléphone). J'imagine que cette photographie est une métaphore du texte.

R. DES ROCHES - Une métaphore due au hasard (je parle du passant). Lucie Ménard savait ce qu'elle faisait lorsqu'elle a suggéré le cadre de cette photographie. Ce passant entre nous deux pourrait - et je dis bien «pourrait» figurer l'autre dont on parle dans ces textes. L'interpellée. Le dénominateur commun. Mais aussi la distance entre les styles, distance qui, pendant un certain temps et par le biais de cette collaboration, ira s'amenuisant. Par échanges de styles, d'effets de style, mes phrases commenceront à ressembler aux siennes et les siennes aux miennes. Pendant un certain temps. Maintenant c'est autre chose. L'effet «de Bellefeuille» a quitté mes textes; même chose pour lui. Et l'effet «de Bellefeuille» a aussi quitté les textes d'autres auteurs qui faisaient dans les mêmes préoccupations: Marcel Labine, Serge Gauthier. Il est clair que Normand a laissé une marque, le temps de quelques livres, sur nos textes. Puis nous nous sommes éparpillés à nouveau, chacun au niveau de son style propre.

V. \& I. - La distance étant pointée, justement, par le préfixe «télé-» (qui signifie «au loin») qu'on retrouve dans «téléphone».

Autre(s) distance(s): je remarque qu'il y a, sur la quatrième de couverture de deux de vos livres (aux extrémités de votre œuvre, à l'époque), la même photographie. D'une part, pour Tous, corps accessoires..., elle est précédée d'un extrait d'un poème en prose du Vierge incendié de Paul-Marie Lapointe ( «Je serai toujours de l'autre côté de la force...»), d'autre part, pour l'Imagination laïque, elle est suivie d'un vers modifié de ce recueil («tous les défauts de l'aventure modeme» qui devient «tous les défauts de l'écriture moderne!»). Peuton associer, par le biais de cette photographie, cet «autre côté» et ces «défauts»? 
R. DES ROCHES - Je serai toujours de l'autre côté de la force parce que j'aurai toujours tous les défauts de l'écriture moderne? Peut-être... André Roy parlerait de l'«irrecevable», disant de mes textes qu'ils sont irrecevables dans ce que je dis et de la façon dont je le dis. On n'écrit pas comme ça!... Irrecevable, peut-être... Je peux le constater: mes œuvres sont peu ou pas répertoriées, peu ou pas critiquées, et je passe toujours à «ça» de recevoir des prix (ça fait pas sérieux, cette dernière remarque!). Je me sens quelque peu dans une position marginale; marginale face à l'establishment littéraire - ou ce qui en reste - des années 60 , marginal face au pseudo-establishment littéraire d'aujourd'hui. Le renom que j'ai, si j'en ai un, s'avère plutôt de l'ordre de l'«intime». Et il est certain qu'en écrivant, qu'en voulant toujours écrire ainsi, à côté, à côté des modes, en faisant dans l'«écriture personnelle» telle que je l'entends, je serai toujours, au niveau littéraire, de l'autre côté de la force - de l'autre côté du miroir -, c'est-à-dire du côté de ce qui n'a pas de contrôle sur l'écriture des autres. N'est-ce pas là où la force - autre que politique et sociale - se joue? Je me sens parfois parent avec les jeunes écrivains de la «relève», mais aussi très étranger: comme je le disais plus tôt, je ne sens pas nécessairement chez eux quelque chose qui va me nourrir (et, en un sens, je crois que c'est relativement normal: on reste chacun dans nos familles) - et je ne sais pas s'ils sentent chez moi quelque chose qui peut les nourrir. Je continue mon cheminement d'écrivain en ajoutant à mon œuvre: ignorant tout, mais tout, ce qui se joue dans la littérature d'aujourd'hui - les théories, les discours, les disputes -, je vois que mes textes maintenant ont cessé de naître d'une conception de l'écriture pour plutôt naître d'une volonté d'écrire, de continuer, de survivre, d'y survivre, tout en me questionnant surtout sur la raison pour laquelle je persiste. Je n'essaie plus de trouver le véritable sens de l'écriture, ou la véritable nature de ce que je fais: poésie, prose, texte, fiction. J'écris. Je laisse aux autres le soin de donner l'étiquette; d'ailleurs, ils pratiquent ce métier mieux que je ne pourrai jamais le pratiquer. S'ils ont raison, ils auront raison. Peu importe après tout: jamais dans l'histoire de l'écriture - romanesque ou poétique - la théorie n'a eu la moindre influence sur les textes de fiction qui se produisaient à la même époque. Tout texte trop collé à autre chose qu'un questionnement légitime et urgent va à sa perte: le nivellement, la banalisation. C.Q.F.D. Si on songe aux énergies que certains ont gaspillées - et gaspillent encore - à vouloir à tout prix illustrer une théorie ou dépendre d'une théorie, on a raison de frémir! Sans demander aux écrivains qu'ils fassent dans le spontané et dans le «tout est valable», on peut demander - et c'est ce que j'essaie de pratiquer - qu'ils placent la pratique de l'écriture avant toute considération de mode (mais en connaissance de cause, dois-je ajouter, car une telle position demande une «expérience» d'écriture, des connaissances pratiques et intellectuelles réelles), qu'ils placent l'écriture devant toute volonté de justification par le théorique. Analysez après. Laissez les autres analyser. Si c'est bon, c'est bon. Si c'est pas bon, c'est pas bon. J'en suis revenu, malheureusement, à certaines notions plutôt conservatrices à propos de l'écriture. Je n'ai peut-être pas raison. Voilà peut-être pourquoi je me sens si souvent «à côté»... Et à voir mes chiffres de vente, je ressens parfois la peur d'être tellement à côté que je demeurerai à jamais le "phénomène Des Roches», comme il y a eu le «phénomène Gauvreau»! 
V. \& I. - Vous n'êtes peut-être pas du côté du pouvoir en littérature, mais vous occupez quand même maintenant une position de pouvoir dans votre travail, alors que ce travail relève aussi du culturel.

R. DES ROCHES - Bien sûr. J'occupe actuellement une «position de pouvoir» chez Logidisque, puisque je fais partie de l'administration de cette compagnie. On pourrait dire, avec raison, que je suis maintenant du côté de la force. D'un certain côté d'une certaine force. La force, une certaine force, la sociale cette fois, est toujours un peu intrigante, séduisante; on la flirte toujours un peu. Et il ne faut pas écraser l'une sur l'autre une position d'écrivain et une position d'administrateur. Ma position d'écrivain demeure aussi précaire qu'aux premiers jours. Ma position d'administrateur ne me donne aucun levier sur l'écriture de mes pairs.

\section{V. \& I. - Quels seraient les défauts de l'écriture moderne?}

R. DES ROCHES - Si je puis me permettre de me défiler rapidement à cette question, je vous ferai remarquer - et honnêtement - que cette phrase est une boutade, une formule qui me semblait, à l'époque, attrayante puisqu'évidemment antithétique.

L'écriture moderne est le défaut de l'écriture moderne.

Si l'on regarde comme animal curieux l'écriture moderne, on peut voir qu'elle contient son propre mécanisme d'extinction. Comme projet, l'écriture moderne - beaucoup plus que l'art moderne qui peut, à la limite, plaire par son aspect décoratif - se révèle d'une absurdité à brailler. Vouloir à tout prix défaire le confortable, jouer avec le doute, déroger, se distinguer - le mouvement est à la fois merveilleux et suicidaire. Bien sûr, l'histoire finira par nous rattraper sinon dans nos textes au moins dans nos «réputations». Jusqu'à ce jour, et dans certaines limites, nous avons encore trop de défauts pour être avalés par l'histoire - et ce, malgré les bourses, les prix, les quelques postes que nous allons un jour occuper.

Les défauts... Je vais réfléchir tout haut... Mon écriture moderne présente les défauts de l'intimisme, du manque d'envergure (je ne parle que pour moi), de la fébrilité (le grain du texte change encore, malgré les années). Elle manque de projet, autre que des projets ponctuels, des images qui s'imposent avec la force d'une idée fixe... L'écriture, chez moi, est un besoin irréfléchi, sans projet précis. J'ai très peu de choses à dire. Du moins, à ce qu'il me semble. Depuis quelques années, mes thèmes se resserrent autour d'une même problématique: l'impossibilité ponctuelle de vivre, de s'entendre à deux, l'impossibilité d'assimiler l'autre, de s'assimiler à l'autre, etc. Tout ce qui est «réflexion» sur l'écriture se résume à des phrases isolées, parfois hors contexte, dont j'émaille mes textes afin de les mieux saisir. Bien sûr, mon écriture est indissociable de ma vie, peu importe le peu de temps que j'arrive à accorder à cette pratique. Mais je viens encore à l'écriture avec le même plaisir, la même appréhension et la même ironie qu'avant. L'ironie, ma marque de commerce! J'ai toujours été ironique dans l'écriture et dans la vraie vie. Je doute toujours de ce que je dis et de ce que je fais. Si je me nomme dans mes textes (mariée qui a les fesses roses et le ventre 
Roger, par exemple, pris dans mon dernier livre), c'est qu'en quelque part, je tiens à réduire l'image de l'auteur qui se permet d'écrire des obscénités sur le dos des autres. De belles obscénités... Le défaut de toute écriture, moderne ou pas, c'est d'être vital pour la seule personne qui la pratique. Si je n'écris pas, je fonctionne mal, très mal. Je ne me sens pas complet. J'ai été trahi. Je vis des deuils qui s'apparentent aux deuils de la rupture. Je continue à réfléchir tout haut... Je vous livre ça en vrac... Je vis ma relation avec l'écriture comme une relation avec une femme. Le besoin en devient physique. Il y a effet de manque infantile... Peu importe la raison, c'est inéluctable, je vais écrire. La dédicace de la seconde partie du Soleil tourne autour de la terre se lit ainsi: $\grave{A}$ François Charron. Il faut écrire.

Un défaut: le manque de souffle et le manque d'idées. Parlons plutôt d'un manque de pouvoir de réflexion. Mes idées me viennent en petits tas, opaques. La forme poétique demeure pour l'instant la seule qui me permette de les «exprimer» d'une façon relativement fidèle. Ces idées sont liées à une perception intuitive de la réalité. L'analyse, ce n'est pas mon fort. Je laisse parfois le texte - l'inconscient, si vous me permettez ce mot bizarre - me mener. Prenons, par exemple, un vers de Tout est normal, tout est terminé. Les mots sont contents, ses seins sont petits, la vie ne continue pas. Il y a une allure surréaliste, puis une allure aphoristique. L'aphorisme entre en collision avec l'effet parapluie et machine à coudre. C'est ma façon à moi d'amener le résultat d'une réflexion ou de faire naître cette réflexion: je m'oblige à penser lorsque j'écris ainsi. Comme lorsque j'écris: C'est la peur d'écrire qui me fait écrire ou encore On n'écrit pas des poèmes, on les corrige.. J'aimerais pousser plus loin ce type de condensation, de réflexion, dans le prochain livre (qui porte déjà un titre: Caur complet). Tout est normal... est exempt d'une bonne partie de la pacotille - de l'appareil éditorial, disiez-vous - qui subsistait encore dans mes autres textes: les parenthèses (il n'y en a que deux ou trois, je pense), les italiques, les notes, les phrases en anglais. C'est beaucoup plus unifié, et ça se centre sur mes deux problèmes existentiels: écrire et aimer. Et j'ose écrire le mot «aimer», ce que je ne faisais jamais avant. Comme j'ose écrire le mot «mort». Et quand j'écris: la vie ne continue pas, c'est, la mort dans l'âme, qu'elle ne continue pas avec quelqu'un d'autre, bien qu'elle continue par ailleurs. Par hasard, actuellement, mes livres ne parlent que de l'échec mais, comme ce qui précède l'échec est une partie de plaisir, une grosse partie de plaisir - plaisir entre sujets et entre objets, car l'autre est un objet, dans le Soleil tourne autour de la Terre, parce que j'en suis évidemment un aussi -, il y a beaucoup de référents sexuels heureux. Ces référents sexuels sont les seuls que je me permette. Lorsqu'il y a constat d'échec, c'est d'échec amoureux dont je parle et non pas d'échec sexuel.

V. \& I. - Vous avez à dessein utilisé le mot «pacotille» pour signifier les effets dont la modernité a usé depuis vingt ans. Faut-il comprendre que la pacotille est un des défauts dont nous parlions?

R. DES ROCHES - Si l'on veut bien prendre ma boutade du départ au sérieux, oui, en effet, cela pourrait en être. L'écriture moderne, l'écriture des an- 
nées 70 surtout, a réussi un tour de force unique je crois dans l'histoire de la littérature: le pari du renouvellement perpétuel. Le «différent à tout prix». Chaque livre se dissociait du précédent. Contrairement aux générations passées, chaque livre s'inscrivait dans un contexte de production massive (tous les auteurs de ma génération ont commencé tôt et ont écrit énormément) et dissociée. Le concept de l'œuvre reposait plus sur la capacité de changer que sur la capacité de durer. Il n'y avait plus d'unité de style, d'un livre à l'autre. Un livre, au pire, deux livres dans un style donné; cela clôt une section, et on saute à autre chose. Quoi d'autre? Cette recherche - on l'appelait recherche et, dans la tête de certains, nous devions porter la blouse blanche du chercheur ou du savant fou! - cette recherche, nous l'avons fait porter par les effets: la ponctuation, la graphie, la déconstruction volontaire de la page, de la phrase et du mot. Il fallait trouver autre chose que «sujet-verbe-complément» (surtout dans des textes sans Sujet!). Nous avons misé sur des freins à la lecture. Afin d'illustrer le modernisme, mais aussi afin de se l'expliquer, de le ressentir. Il n'y aurait rien eu de moderne à écrire à l'époque: On n'écrit pas des poèmes, on les corrige. On aurait peut-être proposé:

Poèmes

(s')écrit pas (à pas)
corrigés $\quad$ car corrigés pas (écrits mais reçus)

D'accord, j'exagère peut-être et je donnerai peut-être aussi l'impression de rejeter toute une série d'expériences qui, tout compte fait, auront servi - et auront été nécessaires - à nous rendre où nous en sommes aujourd'hui. J'aime autant me moquer de nos effets de style que de les regretter. Ce qu'il y a de douteux et ce qu'il y a de dramatique, c'est que certains comptent encore sur ces effets pour se démarquer, pour identifier leur «modernité»...

Mais pour résumer ce que je n'arrive pas à dire... Les défauts de l'écriture moderne. Mes propres défauts.

V. \& I. - Tous les défauts de l'écriture moderne: vous les revendiquez, ils sont intégrés de façon de plus en plus ciblée dans votre écriture.

R. DES ROCHES - Je les revendique et je les tiens à distance. Je ne sais pas. J'essaie de comprendre ce qui me fait écrire. Moi. Aujourd'hui, par dépit ou par fatigue, j'en suis rendu à un chacun-pour-soi relativement confortable. Depuis 1982, j'ai un travail qui m'implique énormément, qui prend de mon temps et de mes énergies, qui me fait oublier toutes les affres de l'écriture qui veut se nommer et les angoisses du clan, les problèmes de savoir à quoi j'appartiens, de dire de quel côté je suis. Je suis redevenu agent libre, et seul: je fais mon travail seul, vous faites votre travail seul, on se lira entre nous lorsque ça nous plaira, et l'histoire dira si l'on s'est trompé. Pendant deux, trois ans, je suis disparu de la place publique littéraire: pas de lecture de poèmes, pas de conférence. Pas de critiques. Mais j'ai œuvré à côté (à côté et en plein dedans): je suis le co-auteur, avec trois programmeurs, d'un système de traitement de texte intitulé l'Écrivain public. J'ai eu une couverture plus fournie sur mon travail d'informaticien en général que sur mon travail d'écrivain! Peu importe. Je reviens, maintenant, à la vie publique, parce que je veux revoir tels hommes et 
telles femmes, pour revivre, presque de l'extérieur, le scintillement d'un lancement (quand le lancement va bien!), par exemple, et non pour échanger de grandes questions sur l'écriture. Les alcools, la bouffe, les rencontres, la drague et tout ce qui entoure cette fête. Et non pour ce que j'allais peut-être y chercher à l'époque: le sentiment d'appartenance à un groupe, d'abord, et, ensuite, le plaisir.

V. \& I. - En 1978, vous publiez les Lèvres de n'importe qui, livre qui me semble important par son utilisation - justement - de la «pacotille».

R. DES ROCHES - Et par son utilisation de l'appareil du roman, sans que cela soit un roman, ou même un récit. Plutôt une «suite thématique». Quant à la couverture, elle propose deux fois huit paires de lèvres, les miennes et celles des sept autres personnes auxquelles «s'adresse» le livre.

V. \& I. - Pour ce livre, vous avez fait imprimer une bande (Ce texte n'est pas un texte juste) qui possède une allure issue d'un mode théorique qui a donné les Poésies de Ducasse ou les Aphorismes de Lichtenberg. Le mot «juste» apparaît déjà dans quelques recueils de la «deuxième partie» de votre œuvre. Dans le Corps certain: «(et corps ne sera pas avec sports mais pavé de bonnes intentions (corrigeons: justes)» et dans la Vie de couple: corps foré d' erreurs (n'ai pas le monopole des idées justes), par exemple.

R. DES ROCHES - Cette phrase est tout à fait le contraire de la phrase plaquée sur la couverture de l'Imagination laïque. Elle n'avait rien d'une boutade, marquée qu'elle était de psychanalyse, de marxisme, fortement teintée par la «nouvelle moralité», donc fortement teintée de mauvaise conscience. Il s'agissait, comme projet de vie, on s'en souvient, de ne pas avoir le monopole de la vérité: on ne faisait pas d'écriture monolithique, de texte-écran, etc., et on voulait du texte pluriel, hors de l'ordre (voire «hors d'ordre»). On ne voulait pas que le texte soit pris comme le point final: ce texte n'est pas le texte qui vient tout dire, analyser, finaliser, qui vient tout trancher. Il y avait aussi une peur de dire les choses. Comme si, à les dire, ces choses pouvaient se révéler tout à coup réactionnaires aux yeux de quelqu'un. Des femmes surtout, à l'époque. Beaucoup de culpabilité dans nos mouvements d'écriture et dans nos gestes de plaisir. Il ne faut pas oublier qu'à l'époque commençait à se dessiner le mouvement de censure «de gauche» que l'on vit aujourd'hui. Nous sortions de la période de free for all libertaire auquel tous et toutes avaient cru. Aujourd'hui, on n'a plus à parler de doute, à faire transparaître la culpabilité dans nos textes (peu importe les justifications et les convictions): on le fait pour nous...

Actuellement, je renverserais la proposition: ce texte n'est pas juste un texte.

Je parlerais d'un autre type de doute, beaucoup plus centré sur ma pratique. Et j'irais plutôt questionner ce qui me fait tourner dans tous les sens: pourquoi ai-je l'impression que j'ai malgré toutes mes prétentions toujours cru que les meilleurs textes devaient être des textes justes, que je cherchais le «vrai texte», que je cherchais la «vraie femme», le «vrai texte» sur la «vraie femme» avec les «vraies idées» que j'étais incapable d'énoncer? Il y a chez moi, face à la moder- 
nité, ce double jeu: d'une part, je ne suis pas théoricien mais je vous dis des choses, d'autre part, j'écris de façon moderne mais j'ai toujours voulu écrire de façon «normale». Ce que j'écris n'est pas «normal» (pourquoi ne suis-je pas capable d'écrire de façon «normalè»?), ce que j'ai vécu ce sont des couples non «normaux» (pourquoi ne suis-je pas capable de me caser?). Le désir, donc, d'une certaine sorte de démission, voilà peut-être le sujet de mon prochain livre. Jusqu'à quel point le fait d'être incapable d'écrire un texte juste — dans mon cas très particulier, il me faut un titre qui me plaise, qui me séduise presque sexuellement avant de commencer à écrire le texte, un peu au hasard au début n'amène pas, justement, par un effet de hasard, une belle modernité? Et quand je dis qu'il y a, derrière la modernité, un besoin d'écrire quelque chose de traditionnel, qu'on n'y arrive pas et qu'on doute par dépit, j'ajoute que je pose cette critique essentiellement sur ma façon d'écrire. À moi. Dans les interstices entre mes livres, dans les dizaines de versions qui mènent, de plus en plus, à ces livres, ce qui revient tout le temps, c'est une espèce de vouloir rentrer dans l'ordre, constamment combattu par mon désir d'écrire autre chose. Jeu de bolo intellectuel: l'anormalité du célibataire versus la normalité, versus ce que j'appelle ironiquement, dans le dernier recueil, «l'Existence parfaite» (mariage, enfants et texte simple). Pour avoir une telle existence, il faut évidemment oublier, faire le vide. Mais le vide se révèle parfois plaisant...

V. \& I. - Je lierais le célibataire, le voyeur (plusieurs versions manuscrites inédites s'intitulent le Voyeur) et le cul, qui est chez vous, me semble-t-il, le nom générique de la sexualité (éléments et gestes constituants). Quelles caractéristiques associez-vous au célibataire - quand je lis ailleurs le titre le Célibataire et son sous-titre Éloge de la facilité?

R. DES ROCHES - Je me fais parfois la réflexion dramatique suivante: $S i$ un jour je ne suis plus célibataire, je ne pourrai plus écrire. Tous mes textes, en effet, avant ou après la lettre, parlent de l'échec amoureux qui mène au célibat, ou du célibat qui mène à l'échec amoureux, le célibat étant une façon de voir les choses centrée sur le regardeur. Ou encore: Si jamais je suis en couple et que cela fonctionne bien, que vais-je écrire?, puisque je n'ai pas le goût d'écrire des livres où tout va bien. De la littérature «heureuse». J'accole spontanément - le royaume de la boutade - célibat et facilité alors que je sais pertinemment que l'état de célibataire n'est pas facile, surtout lorsqu'on pense au jour à jour, à la recherche continuelle (à vide et avide), à la solitude.

La facilité comme le plus court chemin entre deux corps, entre deux idées; la satisfaction passant par une résolution rapide du manque (qui, elle, oblige qu'on taise toutes les différences pendant ce bref moment).

Le célibataire est, vit constamment entre deux couples et cela lui demande une espèce d'inconscience continuelle pour pouvoir ainsi fonctionner, machine à l'intérieur d'autres machines.

L'état de célibat est un état dans lequel je suis, et non vers lequel je tends; le résultat d'une façon (réaliste ou pessimiste) de voir les choses. Si on revient à l'abîme, à la discontinuité de Bataille, on comprend qu'on est fondamentalement séparé de l'autre, qu'on est nécessairement célibataire. L'autre doit demeurer et 
devenir une célibataire dans son rapport avec moi, ce qui n'exclut pas des ententes, des complicités, mais présume surtout du témoignage, un(e) célibataire recherchant un témoin de sa propre vie. L'écrivain fait, par définition, un travail de célibataire, un travail qui implique une exclusion de l'autre. Il n'écrit jamais autrement que seul, et il vit son écriture dans un monde parallèle à tous ses efforts de «vie à deux». Le célibataire, l'écrivain, ne vit que pour lui et rejette ses caresses sur lui-même.

Le cul... Le célibataire voit l'autre dans son corps. Pour son corps. L'autre est loin. Il ne la connaîtra jamais, peu importe les efforts et les effets. Le cul, les seins... Les seins, même lorsqu'ils sont petits, font cette différence catastrophique entre l'homme et la femme vêtus. C'est la chair qui parle fort, plus fort que la pensée, l'objet généreux, découvert et recouvert continuellement dans la rue, l'été, quand le célibataire vit sa chute horizontale sous un parasol Pernod, c'est la chair qui est face à soi, à hauteur des mains ou de la bouche, et qui n'implique pas de position de pouvoir. Mon célibataire a une sexualité préhensile, orale, exacerbée, facile, qui se vit dans le fantasme, dans l'appropriation sans permission - de l'autre: on pourrait, en y pensant assez fort, d'une caresse spontanée, toucher à son corps n'importe où, n'importe quand, avant qu'elle ait eu le temps de dire oui ou de dire non.

Je réfléchis encore à voix haute... Dans mon prochain livre, je veux essayer de «situer» l'autre par rapport à moi-même, le célibatairiser. Une perception de la réalité qui donne l'autre comme trop loin. Et soi, trop près.

V. \& I. - Dans la Promenade du spécialiste, vous dites du corps qu'il est «le spécialiste des rapports difficiles». La peau, enveloppe du corps, est «juste entre» l'intérieur du corps et son inverse, l'extérieur où est l'autre, «juste entre» la tendresse et son inverse, l'irritation. Je lierais cela à babil et, par un jeu de langues: il/elle, à Babel que, dans le Corps certain, vous inversez, via Joyce, en Lebab. Ou encore, sur la couverture de Pourvu que ça ait mon nom, aux deux derniers mots de ce titre, les seuls en retrait, qui sont l'inverse l'un de l'autre. Joue ici quelque chose de l'ordre de la réciprocité.

R. DES ROCHES - Mais c'est une réciprocité qui serait proprement impossible: on ne sait jamais ce que l'autre pense, sent, croit, devine... L'autre est un autre. L'autre, c'est l'autre. Voici moi, voici l'autre. La profusion des mots dans le babil, surface du texte, «peuple des signes lumineux» (sur l'écran du micro-ordinateur), peut être à la fois caressante et irritante. La peau, surface du corps, est ce qui goûte et ce qui sent, ce qui se touche et ce qu'on voudrait déchirer lorsqu'on en arrive au point où l'autre n'a plus de nom, ce qu'on voudrait ouvrir pour voir à l'intérieur si j'y suis. Il est interdit de toucher au texte, et c'est pour ça qu'on a tous les défauts de l'écriture moderne: on touche au texte, on le caresse, on le fait se hérisser, on le change. Interdit, également, de toucher à la peau; malheureusement, on ne peut la changer, la transformer en ce qu'on croit et en ce qui nous est le plus proche. Dans mon fantasme, je peux toucher à l'autre comme je le veux et je peux me laisser toucher comme elle le veut. Objet-objet. Et la peau devient la surface sur laquelle j'essaie de me dessiner pour l'éternité. Mais cela ne fonctionne jamais. Jamais toujours... 
Jamais parfait... Jamais juste...

Dans le rapport amoureux, il y a des moments où, fort heureusement, je perds mon nom et l'autre aussi perd son nom (que je croyais connaître), et où l'on peut se donner complètement à l'effroyable égoïsme du célibataire, cet égoïsme qui fait que le plaisir est un plaisir. On dit: je te touche parce que ça me plaît, tu me touches parce que ça te plaît; tu n'essaies pas de me plaire, mais de me faire plaisir; je n'essaie pas de te plaire, mais de suivre ton désir. Le reste tient du discours amoureux - on fait bâiller ses vêtements, on parle avec ses mains, on caresse, on tient, mais cela demeure du protocole - pour en arriver à la petite mort ou ce qui précède la petite mort, où tous les gestes sont permis lorsqu'on se les permet, lorsque l'autre les permet, lorsque l'autre s'oublie et m'oublie. Comme s'il y avait ceci comme projet: on peut enfin toucher à cette espèce d' «impossible», comment donc va-t-on s'aimer? On va devoir choisir la pornographie, c'est-à-dire la liberté totale et le respect fondamental des différences qui hurlent.

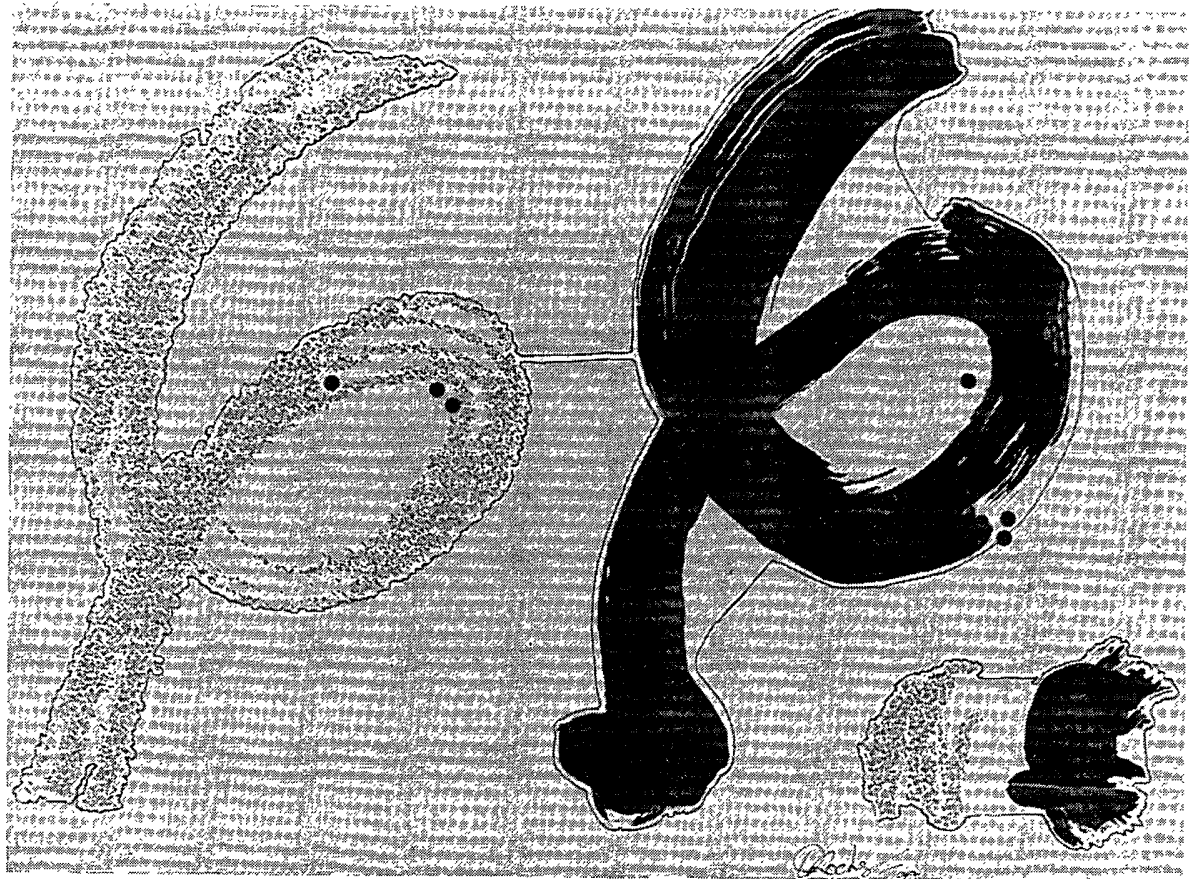

